\title{
Retrospective Analysis of Articular Surface Defect Cases Caused by Pathological Fracture of Giant Cell Tumor of Distal Femur Treated With Three- Dimension-Printed Custom-Made Prostheses Versus Conventional Endoprosthesis Replacement: Three-Dimension-Printed Custom-Made Prostheses Have Better Function Outcome
}

\section{Yuqing Ji}

Department of Orthopaedic Oncology, Qilu Hospital (Qingdao), Cheeloo College of Medicine, Shandong University

Yuxian Wu

Department of Orthopaedic Oncology, Qilu Hospital (Qingdao), Cheeloo College of Medicine, Shandong University

\section{Qiang Yang}

Department of Orthopaedic Oncology, Qilu Hospital, Cheeloo College of Medicine, Shandong University Zhenfeng Li

Department of Orthopaedic Oncology, Qilu Hospital, Cheeloo College of Medicine, Shandong University Jianmin Li ( $\nabla$ bonetumor_ljm@163.com )

Qilu Hospital (Qingdao), Cheeloo College of Medicine, Shandong University https://orcid.org/00000001-8389-8992

\section{Research}

Keywords: Giant cell tumor of bone (GCT), Knee, pathological fracture, 3D-printed prosthesis

Posted Date: June 17th, 2020

DOI: https://doi.org/10.21203/rs.3.rs-34890/v1

License: (c) (i) This work is licensed under a Creative Commons Attribution 4.0 International License. Read Full License 


\section{Abstract}

Background: Pathological fracture of the distal femur giant cell tumor (GCT) occasionally leads to articular surface defect. The conventional endoprosthesis replacement needs to remove the both condyles, and then the patients probably face lower prosthesis survival rate and multiple joint replacement, especially in young patients. The aims of this study were to evaluate the feasibility of 3Dprinted custom-made prosthesis in repairing the articular surface defect at a short-term follow-up.

Methods: We retrospectively reviewed fourteen patients admitted and treated from January 2012 to January 2018 with mean age of 43.9 years (range 29 to 69 years). Diagnoses were primary pathological fracture of distal femur giant cell tumor (GCT) which articular surface of one condyle is defect. 10 lesions were located in medial femoral condyle and 16 in Lateral femoral condyle. Twelve patients were treated with three-dimension-printed custom-made prosthesis to repaired of articular surface defect (3D-printed group), and the other Fourteen patients were treated with endoprosthesis replacement (EPR group). Operation time, blood loss was compared between the groups. Functional results were assessed with MSTS score. The range of motion (ROM), local recurrences and complications were statistically compared.

Results: Mean Follow-up is 33.5 months (range 26 to 43 months). In the 3D-printed group, operation time and blood loss were significantly less $(p<0.05)$. Differences in functional outcomes of the MSTS were observed between the two groups from three-month follow-up $(p<0.05)$ to twenty-four-month follow-up $(p<0.05)$, and the score were higher in the 3D-printed group than the EPR group. The ROM of the 3Dprinted group was statistically better than the EPR group at 6 and 9 months after operation $(p<0.05)$. Local recurrence and complication rates were similar in the two groups $(p=1)$.

Conclusion: 3D-printed custom-made prosthesis represent a promising reconstructive technique in articular surface defect caused by pathological fracture of GCT of distal femur. It could be able to achieve satisfactory oncological and better short-term functional results compared with endoprosthesis replacement. Further studies are needed to evaluate the stability of the implants and the wear of the articular surface with larger samples at long-term.

\section{Background}

Giant cell tumor (GCT) of bone accounts for approximately $5 \%$ of all primary bone tumors[1]. The rate of pathological fracture at presentation in these patients is $4 \%$ to $50 \%[2]$, with $12 \%$ of the fracture located at the distal end of the femur[2]. Pathological fracture of distal femur giant cell tumor (GCT) frequently leads to joint surface defect, which is challenging to be repaired. Due to the eccentric growth of GCT[3], such joint destruction is often confined in one condyle, but conventional methods needs to remove the both condyles together followed endoprosthesis replacement which will face lower prosthesis survival rate and multiple joint replacement[4-6], especially in relatively young patients. So, considering these 
lesions destroy a part of the articular surface, it makes sense to discuss partial repair of the articular surface.

In recent decades, with the development of technology, 3D-printing technology was reported that had enhanced the accuracy and individuation of orthopedic surgeries[7-9]. The use of 3D-printing technology in orthopedic surgery allows greater freedom of prosthetic design and the fabrication of more complex shapes[10], so it is the possibility to reconstruct defects in musculoskeletal oncology with personalized custom-made prostheses[11] . This technology can not only increase the accuracy of the reconstruction, but also reduce the rate of post-operative complications and potentially improving stability[12]. So, it is the possibility to reconstruct defects in musculoskeletal oncology with personalized custom-made prostheses.

At present, as far as we know, there is no report of using 3D printing technology to repair the articular surface defect caused by pathological fracture and comparative analysis with traditional endoprosthesis replacement. We performed a retrospective study. The aims of this study were to evaluate (1) the feasibility of 3D-printed custom-made prosthesis in repairing the articular surface defect caused by pathological fracture of GCT of distal femur and (2) the functional results, complications, and outcomes at short-term follow-up.

\section{Methods}

\section{Patients}

The inclusion criteria of operation were: (1) giant cell tumor of distal femur according to histological evidence; (2) defect of distal articular surface of one side of femoral condyle caused by pathological fracture; (3) the follow-up time is more than 24 months and the follow-up data are complete. The exclusion criteria were (1) non-surgical treatment, (2) combined with cruciate ligament, meniscus, collateral ligament injury or serious joint degeneration, (3) bilateral condylar lesions or multiple articular surface defects, (4) preoperative treatment with denosumab. We retrospectively analyzed our database and identified 26 patients with articular surface defect caused by pathological fracture of GCT of distal femur treated from January 2012 to January 2018.

Data for age, sex, lesion site, Campanacci classification [13], operation time, blood loss, the Musculoskeletal Tumor Society (MSTS) scores[14], local recurrences and complications were collected (Table 1). In total, 12 patients were treated with three-dimension-printed custom-made prostheses (3Dprinted group) (7 males, 5 females; mean age, 42.2 years, range 29-69 years), and 14 patients were treated with endoprosthesis replacement (EPR group) ( 9 males, 5 females; mean age, 45.4 years, range 34-68 years). All surgeries were performed by the same medical team. This study was approved by the by the Ethics Committee of Qilu Hospital of Shandong University Medical Ethics Committee. All patients gave written informed consent to be included in scientific studies at the admission to the hospital.

\section{The design and manufacturing of the 3D-printed custom-made prosthesis}


All the patients were evaluated on preoperative imaging such as Fig. 1(a-d). Not only pre-operative plain radiography and magnetic resonance imaging (MRI) of the distal femur eroded by tumor were evaluated, but also the both distal femurs of the affected side and the healthy side were scanned with computerized tomography (CT, $1 \mathrm{~mm}$ slice intervals). Then, the DICOM format data of CT were transferred to Mimics Medical 21.0 software (Materialise, Ann Arbor, MI). Masks of the both distal femurs were established using the Region Growing command. The 3D models of the both distal femurs were then obtained using the Calculate Part command. The 3D models of the affected side distal femurs before eroded by tumor were established using the mirror command from healthy side. Then, Boolean logic was used to model the defect articular surface from the models before and after pathological fracture. Geomagic studio 12 (Geomagic, Morrisville, NC) was used to help transform the defect articular surface model into a solid model which can be edited by Siemens NX 12.0 software (Siemens PLM Software, Plano, TX). Siemens NX 12.0 software was used to make the defect articular surface $0.5 \mathrm{~mm}$ lower than the normal knee articular surface which is measured according to the MRI. In order to increase the stability of the defect articular surface, screws that pass through the defect articular surface of the prosthesis and an extra plate for fixation of the prosthesis were designed together with engineers using Siemens NX 12.0 software. (Fig. 2). A model of the lesion can be printed used to help understand the condition in more detail before surgery (Fig. 3a-b). Then, the final prosthesis was printed with the electron beam melting (EBM) technology of titanium powder, and the articular surface was highly polished (Fig. 3c-d). It took approximately two weeks for the design and manufacture of each personalized 3D-printed prosthesis (Shanghaishengshi Co., Shanghai, China).

\section{Surgical procedure}

3D-printed group: The surgery was performed under general anesthesia. The tumor was removed by curettage, which is performed through the window of the defect part of the articular surface. If the window of the defect part is not large enough to scrape away all the tumor, an additional window should be opened at the distal femur where was not covered by articular surface. After removing all visible tumor tissues, the tumor cavity is cauterized with electric knife, and then expanded with a high-speed burr (Fig. $3 e$ ). After the curettage was completed, anhydrous alcohol and distilled water pulse irrigation was used to rinse the cavity. Then the tumor cavity was filled with bone cement. The 3D-printed prosthesis should be installed before the bone cement sets. The installation process shall ensure the joint surfaces are in the proper position. (Fig. 3f-h).

EPR group: The surgery was performed under general anesthesia. A median incision in front of the knee joint was used and the distal femur was exposed outside the diseased bone or tumor mass. Osteotomy of the distal femur was performed according to preoperative measurements and A tibial osteotomy was performed in the same manner as a standard knee arthroplasty. A tumor type prosthesis (Beijingchunli Co., Beijing, China) was reconstructed by conventional methods after the resection of tumor.

\section{Post-operative management}


The postoperative management of the two groups was similar. Range of motion exercises of the knee were performed from postoperative day 1 . The patient was allowed non-weight-bearing standing and walking with 7 days after surgery. Partial weight-bearing with crutches was encouraged from 2 weeks postoperatively, followed by gradual full weight-bearing. The patient was followed up every month for the first 3 months, then every 3 months thereafter. The oncology outcomes, and radiographs were assessed at each follow-up.

\section{Statistical analysis}

Continuous data are expressed as mean \pm standard deviation. The independent sample t test were used to compare the age, operation time, bleeding volume, ROM and MSTS score of the two groups. The $x 2$ test was used to analyze the sex, tumor location, postoperative complications and recurrence rate of the two groups. A p-value of $<0.05$ was considered to be significant. Statistical analysis was performed using the SPSS Statistics software version 22.0 (IBM, Armonk, NY, USA).

\section{Results}

\section{Operational outcomes}

All operations were performed successfully and no intraoperative complications occurred. The operating time in the 3D-printed group was (123.3 \pm 20.7$)$ minutes (IQR 100-160minutes) , compared with the operating time in the EPR group of (146.4 \pm 18.8$)$ minutes (IQR 120-170minutes). The blood loss during

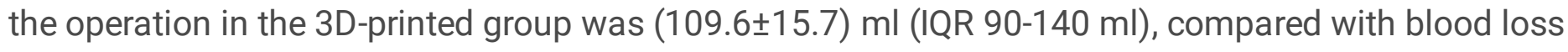
during the operation in the EPR group of (182.1 \pm 31.9$)$ minutes (IQR 130-225minutes). The operating time and the blood loss were significantly less in the 3D-printed group $(p<0.05$ and $p<0.05$, respectively).

\section{Oncological outcomes}

The mean follow-up was for 33.5 months ranging from 26 to 43 months. One patient in the EPR group experienced postoperative recurrence, which was a female patient. The recurrence time was 9 months after the operation and the patient underwent a second resection. All the other patients were disease-free during the follow-up period.

\section{Post-operative functional status}

The recovery of the affected limb function was assessed according to the MSTS score. The MSTS function score of the two groups improved continuously after operation, but the score improved of the 3D-printed group was better than the EPR group. (Fig. 4a). The 3D-printed group had a mean score of 23.0 (18 to 27) at 3 months after operation while had a mean score of 28.2 (27 to 30 ) at 24 months after operation (Fig. 5a-d). The EPR group had a mean score of 20.1 (17 to 24 ) at 3 months after operation while had a mean score of 26.2 (25 to 28 ) at 24 months after operation. The difference was statistically significant ( 3 months after operation: $\mathrm{T}=2.518, \mathrm{P}=0.019 ; 12$ months after operation: $\mathrm{T}=4.227, \mathrm{P}=$ $0.000 ; 24$ months after operation: $T=5.433, P=0.000$ ). The ROM of the 3D-printed group was 
statistically better than the EPR group at 6 and 9 months after operation $(p<0.05)$, but there was no significant difference in ROM between the two groups from 1 to 2 years of follow-up. (Fig. 4b) .

Complications.

3D-printed group: One patient had delayed wound healing and then the wound healed after dressing change without the second operation. No patient had a deep infection. Postoperative radiographic examinations showed no evidence of bone resorption or osteolysis at the prosthesis-bone interface. There was no heterotopic ossification, loosening, breakage or displacement of the 3D-printed prosthesis. The EPR group: There were 3 patients with postoperative incision complications ( 1 male, 2 females, considered to be related to obesity and postoperative low albumin). All patients healed 2 weeks after operation debridement and catheter flushing. One case of prosthesis loosening occurred 26 months after the operation, and a prosthesis revision was performed.

\section{Discussion}

In this study, we used the 3D printing technology to help restore the digital model of the anatomic morphology before lesion destruction by using mirror digital of the healthy side of the body. We found that the surgical procedures performed in the 3D-printed group were shorter in operating time and less in blood loss during the operation compared with the EPR group, and the average dropped by $15.8 \%$ and $39.8 \%$ respectively. The values dropped of operating time and bleeding amount indicate that the surgical procedure of the 3D-printed group is less traumatic and complicated. In our study, the mean MSTS score in the 3D-printed group was 28.2 ranging from 27 to 30 is better than 26.2 ranging from 25 to 28 in the EPR group at 24 months after operation, and the mean MSTS score in the 3D-printed group also is better than the ranged from 24.5 to 26 in those treated with resection reported previously $[15,16]$. This is maybe because of the less traumatic and the use of bone cement that allow patients to exercise early. In 3Dprinted group, no composite fracture or prosthesis loosening was found which is similar as reported that curettage could avoid the increased complications associated with wide resection[16, 17].

The difference between curettage and wide resection is always one of the hotspots in the treatment of GCT of bone with pathological fracture[16, 18]. Recent studies have shown that curettage can achieve local tumor control rates similar to resection while reducing the incidence of complications[2]. At the same time, other recent studies have also shown that pathological fractures are not a risk factor for recurrence, and the presence of a pathological fracture should not preclude the decision to perform curettage[18]. A meta-analysis showed that it has difference in local recurrence rates, but the difference in the risk of local recurrence did not reach statistical significance in the wide resection group compared to curettage group[19], and the meta-analysis suggest the presence of a pathological fracture should not be only influential factor in the decision making to perform wide resection or curettage. In our study, all patients in 3D-printed group underwent curettage before reconstruction of the articular surface, and satisfactory tumor control was achieved during the mean follow-up compared with the EPR group. 
If a curettage procedure is chosen, in addition to carefully intraoperative tumor scraping, adjuvant therapy is essential[16], which is important to reduce tumor recurrence. After the tumor is scraped off during surgery, Local adjuvant therapy (argon beam coagulation, phenol, ethanol, hydrogen peroxide, liquid nitrogen, etc.) is often used in the treatment of residual tumors in the lesion[20]. In 3D-printed group, the adjuvant therapy was combined with electric knives, grinding drills, anhydrous alcohol and filled with bone cement. Although there are many pre-operative studies using denosumab[21], we had not used them, because some recent literature suggests that the use of denosumab before curettage operation may result in a higher recurrence rate because tumor cells are easily retained in the operation[22, 23].

In the 3D-printed group, the surface repair of the joint is made of titanium alloy metal material. Although the individualized bionic design and high polishing procedure are used, the wear of the corresponding tibial articular surface is theoretically larger than the original joint surface, as it is usually believed can accelerate the degeneration of the joint. But during our follow-up, there was no significant difference in the medial and lateral space of the joint in the patients of 3D-printed group (X-ray film taken while standing). So, the wear of the corresponding tibial articular surface may be not as fast as it was generally thought in the past. However, even this happens in the end, it delays the use of non-conventional endoprostheses replacement and reduce the number of revision surgery. Considering that traditional prosthetic revision surgery is often accompanied by lower bone mass and high failure rates[24], the surface repair surgery using three-dimension-printed custom-made prostheses should benefit the patients.

The discussion of some surgical treatment should be clear about the application boundary. The surface repair surgery using three-dimension-printed custom-made prostheses are also obviously not suitable for all patients with articular surface defects caused by pathological fractures of GCT of the distal femur. In our experience, some patients whose joint surfaces of both femoral condyles have articular defects are more suitable for tumor-type prosthesis replacement than surface repair surgery using three-dimensionprinted custom-made prostheses. Some patients whose injury involving the cruciate ligament or collateral ligament are not recommended to use surface repair surgery by three-dimension-printed custom-made prostheses, especially in the patients whose ligaments reconstruction are difficult. Some elderly patients, whose articular surface degeneration is heavier, are more suitable for tumor-type prosthesis replacement surgery rather than articular surface repaired surgery described in this article.

The articular surface repaired surgery used three-dimension-printed custom-made prostheses should be cautious in the implementation process and should be controlled in all aspects, especially in the following aspects: 1 . The design of the articular surface and the subsequent high polishing must be precise, which are the keys to reduce the joint degeneration of patients. 2 . The elastic modulus of the metal articular surface and the normal articular surface is different, so according to our experience, the metal articular surface should be $0.5 \mathrm{~mm}$ lower than the normal articular surface during design, in order to make the stress of the entire joint more balanced. 3. The plate added to the articular surface prosthesis should also be designed according to the individualized bone of the patient using 3D printing technology. A well-designed plate can not only increase the stability of the articular surface prosthesis, but also 
provide additional guidance for the implantation of the articular surface prosthesis because of its good matching with the patient's bone. 4. The inner side of the articular prosthesis can be designed with protrusions or porous structure[25] to better grip the bone cement and increase stability.

The main limitation of this study is the relatively short follow-up. Loosening and fracture of implants might not occur until several years after the operation. Assessment of the wear of the prosthesis and the corresponding tibial articular surface in the long-term will also be required. The survival and oncological outcomes will also need to be recorded with longer follow-up.

\section{Conclusions}

The use of 3D-printed titanium prostheses is growing in musculoskeletal oncology. 3D-printed custommade prostheses represent a promising reconstructive technique in articular surface defect caused by pathological fracture of GCT of distal femur. It could be able to achieve satisfactory oncological and good short-term functional results. Further studies are needed to evaluate the stability of the implants and the wear of the articular surface at long-term.

\section{List Of Abbreviations}

GCT: giant cell tumor

ROM: range of motion

MSTS: Musculoskeletal Tumor Society scores

\section{Declarations}

Ethics approval and consent to participate: This study was performed in line with the principles of the Declaration of Helsinki. Approval was granted by the Ethics Committee of Qilu Hospital of Shandong University Medical Ethics Committee (December 28, 2015/No. 2015087).

Consent for publication: Written informed consent was obtained from the patient for publication of this article and accompanying images.

Availability of data and materials: The datasets supporting the conclusions of the article are included within the article.

Competing interests: The authors declare that they have no competing interests.

Funding: This study was funded by a governmental foundation, Qingdao Key Health Discipline Development Fund (No. QDZDXK-A-2017005). 
Acknowledgements: The authors gratefully acknowledge the staff in the Department of Orthopaedic Oncology, Qilu Hospital (Qingdao), Cheeloo College of Medicine, Shandong University.

Author contributions: Yuqing Ji, Yuxian Wu, and Jianmin Li were involved with the concept and design of this manuscript. Qiang Yang and Zhenfeng Li were involved with the acquisition of subjects and data. Yuqing $\mathrm{Ji}$ and Jianmin Li were involved in the design of the prosthesis. Yuxian Wu were involved in postsurgical evaluation of the patients. All authors contributed toward data analysis, drafting and critically revising the paper, gave final approval of the version to be published, and agree to be accountable for all aspects of the work.

\section{References}

1. Xu SF, Adams B, Yu XC, Xu M: Denosumab and giant cell tumour of bone-a review and future management considerations. Curr Oncol 2013, 20:e442-447.

2. Tsukamoto S, Mavrogenis AF, Tanzi P, Leone G, Righi A, Akahane M, Kido A, Honoki K, Tanaka Y, Donati DM, Errani C: Similar local recurrence but better function with curettage versus resection for bone giant cell tumor and pathological fracture at presentation. J Surg Oncol 2019.

3. van der Heijden L, Dijkstra PD, van de Sande MA, Kroep JR, Nout RA, van Rijswijk CS, Bovee JV, Hogendoorn PC, Gelderblom H: The clinical approach toward giant cell tumor of bone. Oncologist 2014, 19:550-561.

4. Rigollino AV, Fernando TS, Tanaka MH, Souza MM: Giant cell tumor locally advanced around the knee: treatment and literature review. Rev Bras Ortop 2017, 52:473-478.

5. Barut N, Anract P, Babinet A, Biau D: Peri-prosthetic fractures around tumor endoprostheses: a retrospective analysis of eighteen cases. Int Orthop 2015, 39:1851-1856.

6. Xu S, Yu X, Xu M, Fu Z: Inactivated autograft-prosthesis composite has a role for grade III giant cell tumor of bone around the knee. BMC Musculoskelet Disord 2013, 14:319.

7. Wang KC, Jones A, Kambhampati S, Gilotra MN, Liacouras PC, Stuelke S, Shiu B, Leong N, Hasan SA, Siegel EL: CT-Based 3D Printing of the Glenoid Prior to Shoulder Arthroplasty: Bony Morphology and Model Evaluation. J Digit Imaging 2019.

8. Park JW, Kang HG, Lim KM, Park DW, Kim JH, Kim HS: Bone tumor resection guide using threedimensional printing for limb salvage surgery. J Surg Oncol 2018, 118:898-905.

9. Han Q, Zhao X, Wang C, Chen B, Wang X, Zhang Z, Zhang K, Zheng Y, Wang J: Individualized reconstruction for severe periprosthetic fractures around the tumor prosthesis of knee under assistance of 3D printing technology: A case report. Medicine (Baltimore) 2018, 97:e12726.

10. Wei R, Guo W, Yang R, Tang X, Yang Y, Ji T, Liang H: Reconstruction of the pelvic ring after total en bloc sacrectomy using a 3D-printed sacral endoprosthesis with reestablishment of spinopelvic stability: a retrospective comparative study. Bone Joint $J$ 2019, 101-b:880-888.

11. Angelini A, Trovarelli G, Berizzi A, Pala E, Breda A, Ruggieri P: Three-dimension-printed custom-made prosthetic reconstructions: from revision surgery to oncologic reconstructions. Int Orthop 2019, 
43:123-132.

12. Ran Q, Yang W, Hu Y, Shen X, Yu Y, Xiang Y, Cai K: Osteogenesis of 3D printed porous Ti6Al4V implants with different pore sizes. J Mech Behav Biomed Mater 2018, 84:1-11.

13. Campanacci M, Baldini N, Boriani S, Sudanese A: Giant-cell tumor of bone. J Bone Joint Surg Am 1987, 69:106-114.

14. Enneking WF, Dunham W, Gebhardt MC, Malawar M, Pritchard DJ: A system for the functional evaluation of reconstructive procedures after surgical treatment of tumors of the musculoskeletal system. Clin Orthop Relat Res 1993:241-246.

15. Niu X, Zhang Q, Hao L, Ding Y, Li Y, Xu H, Liu W: Giant cell tumor of the extremity: retrospective analysis of 621 Chinese patients from one institution. J Bone Joint Surg Am 2012, 94:461-467.

16. van der Heijden L, Dijkstra PD, Campanacci DA, Gibbons CL, van de Sande MA: Giant cell tumor with pathologic fracture: should we curette or resect? Clin Orthop Relat Res 2013, 471:820-829.

17. Xing R, Yang J, Kong Q, Tu C, Zhou Y, Duan H: Giant cell tumour of bone in the appendicular skeleton: an analysis of 276 cases. Acta Orthop Belg 2013, 79:731-737.

18. Salunke AA, Chen Y, Chen X, Tan JH, Singh G, Tai BC, Khin LW, Puhaindran ME: Does pathological fracture affect the rate of local recurrence in patients with a giant cell tumour of bone?: a metaanalysis. Bone Joint J 2015, 97-b:1566-1571.

19. Salunke AA, Pathak S, Shah J, Pandit J, Naneria V: Wide resection versus curettage in giant cell tumor with pathological fracture? A systematic review and meta-analysis. J Clin Orthop Trauma 2018, 9:S15-S18.

20. Greenberg DD, Lee FY: Bisphosphonate-loaded Bone Cement as a Local Adjuvant Therapy for Giant Cell Tumor of Bone: A 1 to 12-Year Follow-up Study. Am J Clin Oncol 2019, 42:231-237.

21. Yang Y, Li Y, Liu W, Xu H, Niu X: A nonrandomized controlled study of sacral giant cell tumors with preoperative treatment of denosumab. Medicine (Baltimore) 2018, 97:e13139.

22. Errani C, Tsukamoto S, Leone G, Righi A, Akahane M, Tanaka Y, Donati DM: Denosumab May Increase the Risk of Local Recurrence in Patients with Giant-Cell Tumor of Bone Treated with Curettage. J Bone Joint Surg Am 2018, 100:496-504.

23. Scoccianti G, Totti F, Scorianz M, Baldi G, Roselli G, Beltrami G, Franchi A, Capanna R, Campanacci DA: Preoperative Denosumab With Curettage and Cryotherapy in Giant Cell Tumor of Bone: Is There an Increased Risk of Local Recurrence? Clin Orthop Relat Res 2018, 476:1783-1790.

24. Sevelda F, Waldstein W, Panotopoulos J, Stihsen C, Kaider A, Funovics PT, Windhager R: Survival, failure modes and function of combined distal femur and proximal tibia reconstruction following tumor resection. Eur J Surg Oncol 2017, 43:416-422.

25. Liang H, Ji T, Zhang Y, Wang Y, Guo W: Reconstruction with 3D-printed pelvic endoprostheses after resection of a pelvic tumour. Bone Joint $J$ 2017, 99-b:267-275.

\section{Table}


Table I. Demographic, oncological data of patients $(n=26)$

\begin{tabular}{|c|c|c|c|}
\hline Variables & 3D-printed group $(n=12)$ & EPR group $(n=14)$ & $p$ value \\
\hline \multicolumn{4}{|l|}{ Demographic data } \\
\hline \multicolumn{4}{|l|}{ Gender, n (\%) } \\
\hline Male & $7(58.3)$ & $9(64.3)$ & 0.756 \\
\hline Female & $5(41.7)$ & $5(35.7)$ & \\
\hline Age, mean (SD) yrs & 42.2(SD 11.4) & 45.4(SD 9.6) & 0.437 \\
\hline \multicolumn{4}{|l|}{ Oncological data } \\
\hline \multicolumn{4}{|l|}{ Site, n (\%) } \\
\hline medial femoral condyle & $5(41.7)$ & $6(42.9)$ & 0.951 \\
\hline Lateral femoral condyle & $7(58.3)$ & $8(57.1)$ & \\
\hline Campanacci classification, n (\%) & & & 0.756 \\
\hline I & $0(0)$ & $0(0)$ & \\
\hline II & $7(58.3)$ & $9(64.3)$ & \\
\hline III & $5(41.7)$ & $5(35.7)$ & 0.006 \\
\hline Operating time, mean (SD) minutes & $123.3(20.7)$ & $146.4(18.8)$ & 0.000 \\
\hline Blood loss, mean (SD) ml & 109.6(15.7) & $182.1(31.9)$ & 0.019 \\
\hline MSTS & $23.0(3.1)$ & $20.1(2.7)$ & 0.000 \\
\hline 3 months after operation, mean (SD) & $26.7(1.2)$ & $24.6(1.3)$ & 0.000 \\
\hline 12 months after operation, mean (SD) & $28.2(0.8)$ & $26.2(1.0)$ & 1 \\
\hline 24 months after operation, mean (SD) & $0(0)$ & $1(7)$ & 0.330 \\
\hline Local recurrence, $\mathrm{n}(\%)$ & $1(8.3)$ & $4(28.6)$ & \\
\hline Postoperative complications, $\mathrm{n}(\%)$ & & & \\
\hline
\end{tabular}

Abbreviation: MSTS, Musculoskeletal Tumor Society scores.

\section{Figures}



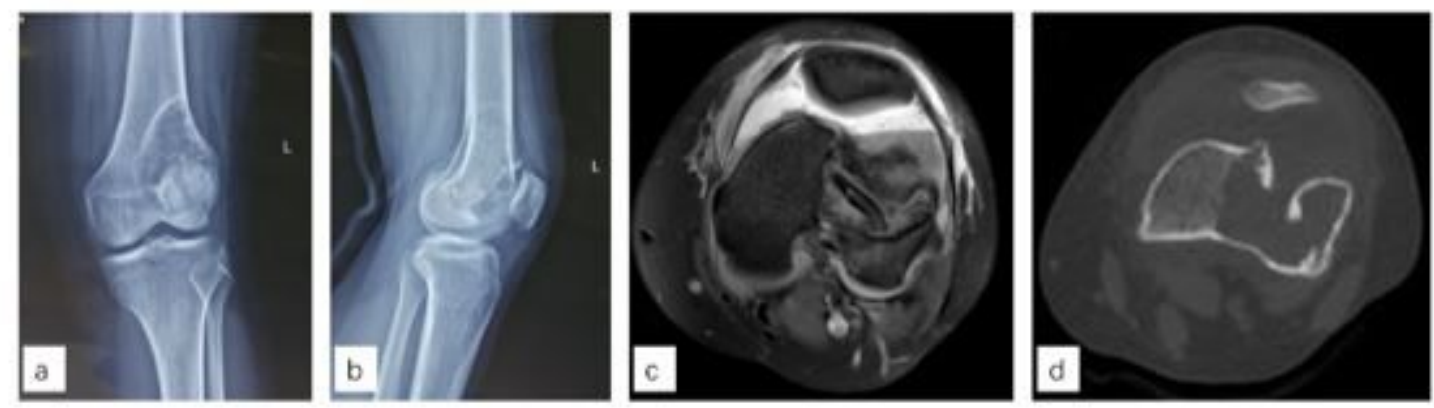

\section{Figure 1}

Imaging examinations of a woman aged 29 years. (a and b) The pre-operative radiographs show that there is pathological fracture of giant cell tumor in the left distal femur. (c) The MRI scans and (d) The CT scans show a articular surface defect was caused by the pathological fracture.

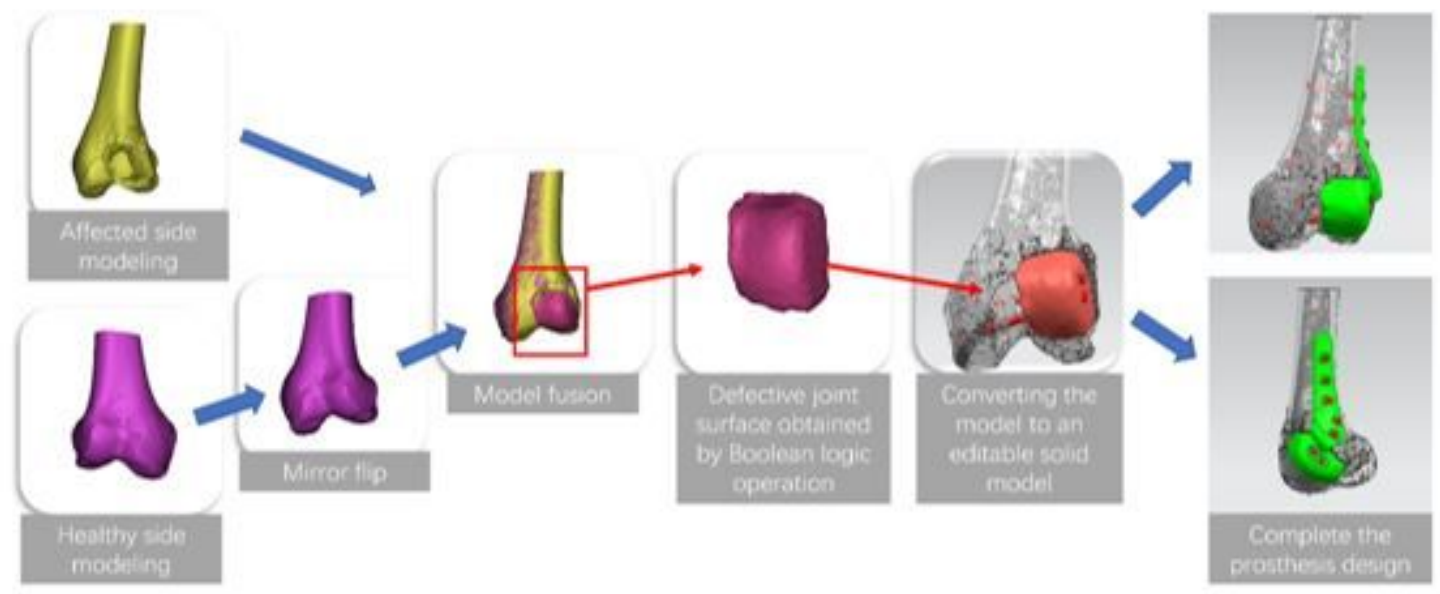

Figure 2

Flow chart of the design of the 3D-printed custom-made prosthesis. 

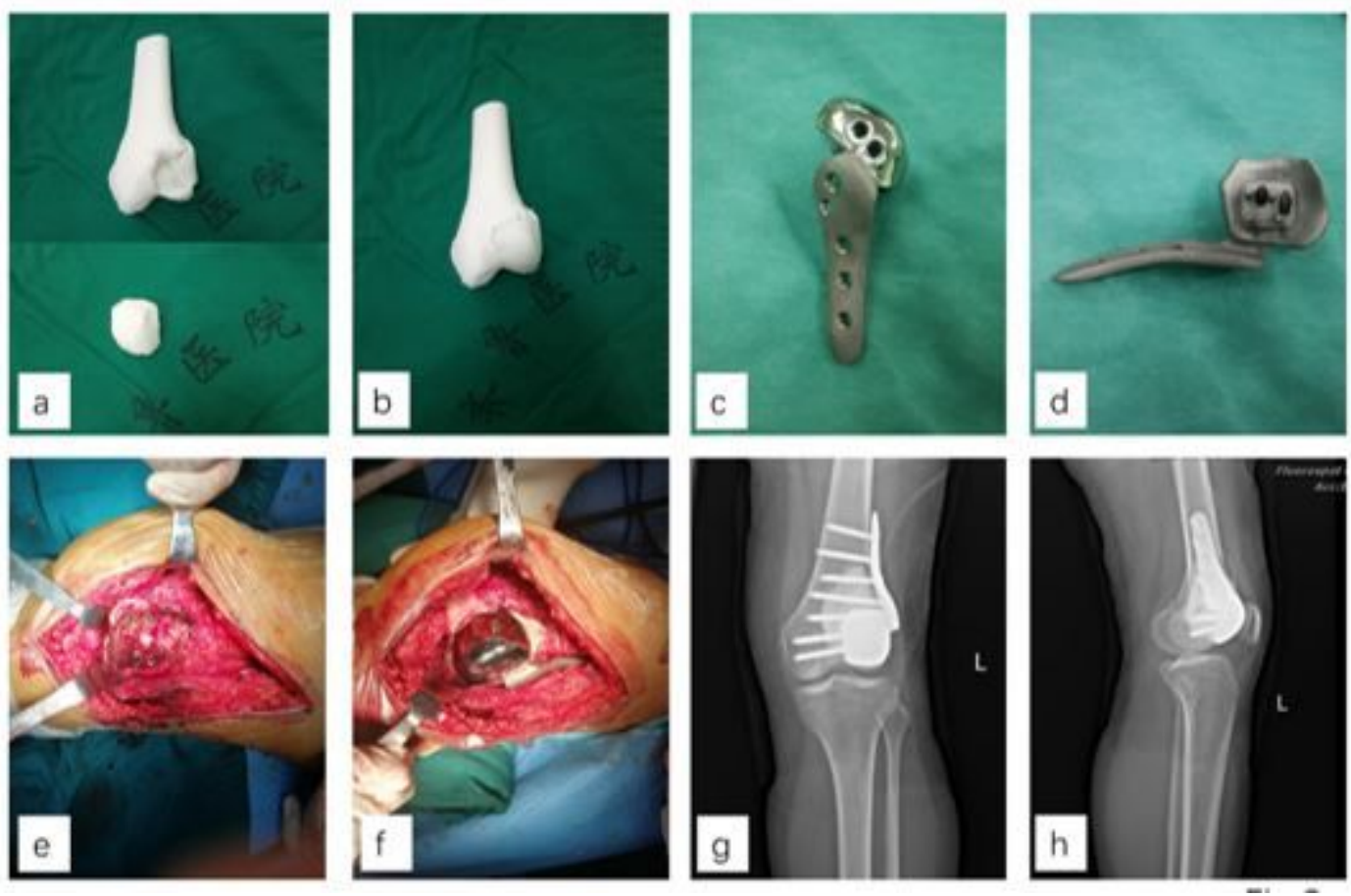

\section{Figure 3}

The 3D printed prosthesis and surgical procedures (a) 3D printed models of the distal femur that has defection of articular surface caused by pathological fracture of giant cell tumor. (b) The defection of articular surface. (c-d) The 3D printed prosthesis. (e) Intraoperative pictures show the tumor is scraped off. (f) The distal femur that defection of articular surface is restored by 3D printed prosthesis and the prosthesis is installed and fixed. (g-h) Postoperative radiographs of the distal femur.
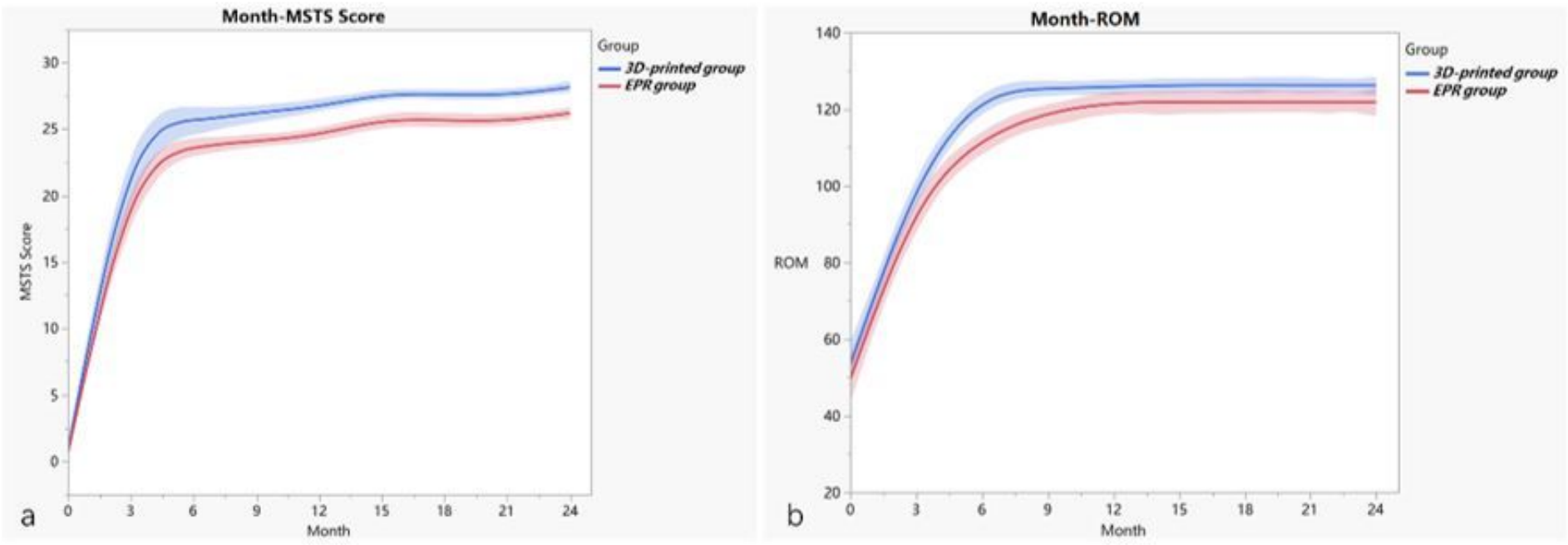

Figure 4

The MSTS function score and ROM after operation. (a) The MSTS function score of both groups was improved continuously after operation, but the score improved of the 3D-printed group was statistically better than the EPR group from three-month follow-up to twenty-four-month after operation. (b) The ROM 
of the 3D-printed group was better than the EPR group, and the difference was statistically at 6 and 9 months after operation.
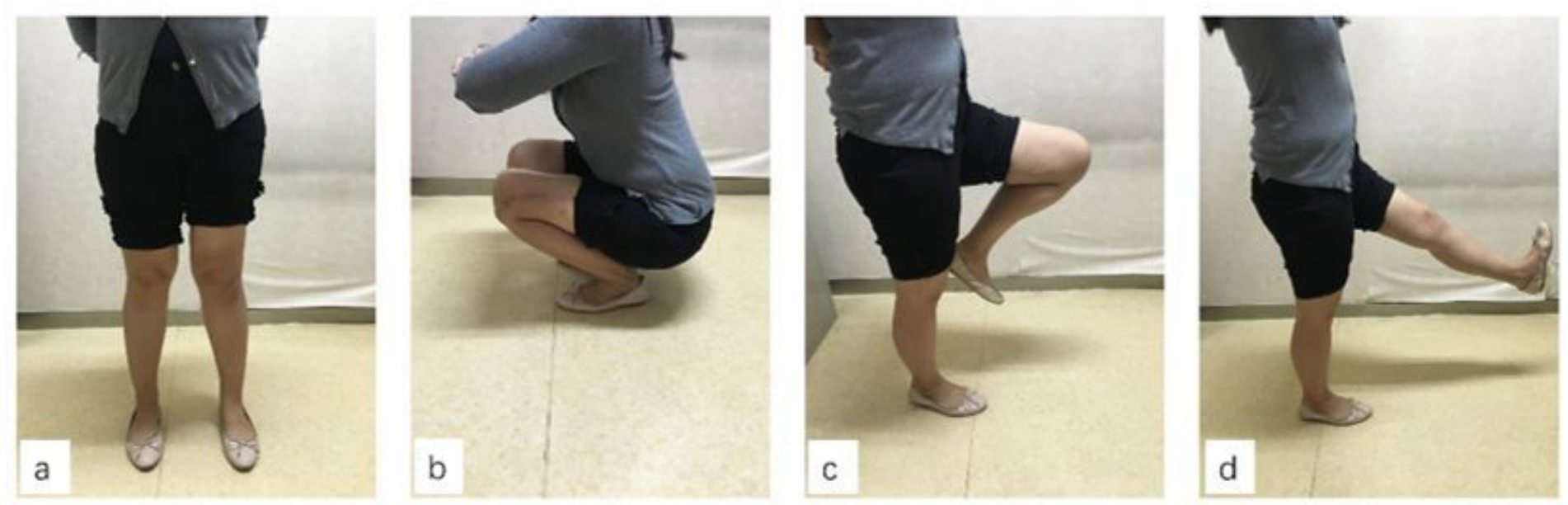

\section{Figure 5}

Postoperative recovery of limb function. Satisfactory limb function 26.2 months after 3D-printed prosthesis reconstruction. (a) Standing. (b) Crouching. (c) Flexion of the knee. (d) Extending of the knee. 